\title{
Link Adaptation and SINR errors in Practical Multicast Multibeam Satellite Systems with Linear Precoding
}

\author{
Anxo Tato $^{1 *}$ | Stefano Andrenacci ${ }^{2 \dagger}$ | Eva Lagunas ${ }^{3 \ddagger}$ | \\ Symeon Chatzinotas $^{3 \ddagger}$ | Carlos Mosquera ${ }^{1 *}$
}

${ }^{1}$ atlanTTic Research Center, University of Vigo, Galicia, Spain

${ }^{2}$ SES, Luxembourg

${ }^{3}$ Interdisciplinary Centre for Security, Reliability and Trust (SnT), University of Luxembourg

\section{Correspondence}

Anxo Tato Arias

atlanTTic Research Center

University of Vigo, Galicia, Spain

Email: anxotato@gts.uvigo.es

\section{Funding information}

This work was funded by the Xunta de Galicia under a predoctoral scholarship (co-funded by the European Social Fund) and it was partially funded by the Agencia Estatal de Investigación (Spain) and the European Regional Development Fund (ERDF) under project MYRADA (TEC2016-75103-C2-2-R). Part of the research was done during a stay at the SnT, University of Luxembourg, supported by the project SatNEx-IV, co-funded by the European Space Agency (ESA). The view expressed herein can in no way be taken to reflect the official opinion of ESA

Note: This is an extended version of the article "Link Adaptation and Carriers Detection Errors in Multibeam Satellite Systems with Linear Precoding", [1].
The application of linear precoding at the gateway side enables broadband multibeam satellite systems to use more aggressive frequency reuse patterns increasing the overall capacity of future High Throughput Satellites (HTS). In any practical precoded system, receivers can estimate only a few coefficients of the Channel State Information (CSI), while the others, in what is known as nullification, are set to zero. In this paper, the impact of the CSI nullification to the SINR estimation is analysed statistically and geographically for a multicast multibeam satellite system. The errors in the SINR calculated by the gateway affect to the Modulation and Coding Schemes (MCS) selection, increasing the rate of erroneous frames or leading to an under-utilization of the available resources. Therefore, as countermeasure, a link adaptation algorithm based on an adaptive margin per user is proposed, helping to achieve the error rate constraints of DVB-S2X systems without compromising the throughput, even under severe CSI degradation due to nullification and Rician fading.

KEYWORDS

satellite communications, multi-beam satellite, precoding, link adaptation, 


\section{1 | INTRODUCTION}

The use of more aggressive frequency reuse patterns, as full-frequency reuse, allows to increase the capacity of the forward link of multibeam satellite communications systems. This approach is proposed to increase the system capacity of High Throughput Satellites (HTS) operating at Ka-band. Linear precoding techniques reveal as a promising method to mitigate the higher co-channel interference which arises in this scenario. Indeed, the new superframe format of DVB-S2X [2] enables the use of precoding in DVB broadband satellite systems.

Several works propose the application of linear precoding to the forward link of DVB-S2(X) based systems, as [3], [4], [5], [6] and [7]. Performance analysis and practical issues are addressed; among others, multiplexing of several users per frame, the importance of users scheduling in the final system capacity, the outdated CSIT (Channel State Information at the Transmitter), or CSI estimation errors.

However, there is an important problem related with imperfect CSI in precoding which previous works do not consider, referred to as the nullification effect. To the best of our knowledge this problem is only partially covered in [8] and it is addressed with much more detail and for more realistic assumptions in this work. In precoded multibeam satellite systems, the CSI of each user is a vector comprising the link response of the intended and interfering beams. It often happens that receivers are not able to estimate many of these channels since the interfering signals from other beams are received with a very low power level. Therefore, the CSI available at the gateway is a sparse vector with many zero entries. This causes two problems. On the one hand, the calculated precoding matrix is not optimized for the actual channel but for the nullified version of the channel matrix, leading to some performance loss. And, more importantly, the Signal to Interference and Noise Ratio (SINR) calculated by the gateway for each user differs from the actual value. The error in the estimation of the users SINR by the gateway can be high enough to lead, in the case of overestimation, to increase the rate of erroneous frames over the QEF (Quasi Error Free) target of DVB-S2(X) systems, since the estimated SINR is used to perform Modulation and Coding Scheme (MCS or MODCOD) allocation.

This work tries to fill the gap related to the nullification error problem found in the literature, explaining how nullification occurs, characterizing the statistical and geographical distribution of the SINR errors due to the nullification effect, and proposing a method to overcome this problem. Since the nullification effect causes wrong MCS allocation due to the errors in the calculated SINR, a link adaptation algorithm with an adaptive margin is proposed as a countermeasure for those frame errors that the nullification triggers. The improved Outer Loop Link Adaptation (OLLA) algorithm described in [9] is used here, instead of the classic OLLA algorithm employed in [1]. Moreover, we quantify in terms of throughput loss the impact of three different countermeasures for the problem of CSI nullification CSI: a global, a beam-based and a per-user backoff margin. Based on the study carried out in this paper, our proposal for solving this issue with the minimum impact in the system throughput is to use a link adaptation algorithm with an independent adaptive margin per user.

In this paper, realistic assumptions are made in order to simulate a system closer to real practical systems. In particular, we include multicast, a basic user scheduling algorithm and Rician fading. In our simulations we address mainly $M=2$ users per frame, a number which may seem small compared with previous precoding papers, but which is in line with the smaller frames proposed for next DVB-S2X precoding waveforms [10]. Here, two different levels of user scheduling are considered, namely, a geographical inter-beam scheduling, which results from dividing all the beams in sectors, and an intra-beam scheduling, by creating multicast groups of $M$ users in each beam based in the similarity of their channel vectors. Furthermore, in the evaluation of the link adaptation algorithm three scenarios are studied: one without fading, as in [1], and other two with Rician fading and different Rice factors, one for terrestrial and other for aeronautical applications.

This paper is structured as follows. Section 2 presents the equations of the system model, describes the multibeam 
channel model, the computation of the precoding matrix and precoded SINRs and also the scheduling using in the simulations. Then, Section 3 explains the different types of CSI carriers nullification and how this effect is modeled. Afterwards, Section 4 presents the system parameters and highlights the importance of the nullification before the main results of the paper on the analysis of the SINR error due to nullification are collected in Section 5. Then, in Section 6 the impact in the throughput of different types of margins is commented. Lastly, before the conclusions, Section 7 describes the link adaptation algorithm used to overcome the problems caused by the nullification, and provides some simulation results showing how the proposed strategy solves this problem.

\section{2 | SYSTEM MODEL}

We consider the forward link of a multibeam satellite communications system with full frequency reuse operating at Ka-band. Linear precoding is used to reduce the co-channel interference which arises in this configuration, significantly higher than that in four-color frequency/polarization configurations [5]. We assume that the satellite operates $N$ beams with a single feed per beam (SFPB) payload. In each frame, data for $M$ users located in the same beam are multiplexed. Therefore, $N \times M$ users are served simultaneously during the duration of a frame, $M$ per beam. With all these considerations the general model for signals received at the User Terminals (UTs) at a given time instant in matrix notation is

$$
\mathbf{y}^{[i]}=\mathbf{H}^{[i]} \mathbf{x}+\mathbf{n}^{[i]}=\mathbf{H}^{[i]} \mathbf{W} \mathbf{s}+\mathbf{n}^{[i]}, \quad i=1,2, \ldots, \mathbf{M},
$$

where the vector $\mathbf{y}^{[i]} \in \mathbb{C}^{N}$ collects the received signals by the $i$-th set of users in the $N$ beams. $\mathbf{s} \in \mathbb{C}^{N}$ are the information symbols for the users, which are normalized in terms of energy, $\mathbb{E}\left\{\left|s_{k}\right|^{2}\right\}=1, \mathbf{x} \in \mathbb{C}^{N}$ are the precoded transmitted signals by each antenna, $\mathbf{W} \in \mathbb{C}^{N \times N}$ is the precoding matrix, $\mathbf{H}^{[i]} \in \mathbb{C}^{N \times N}$ is the channel matrix for $i-$ th set of users in all the beams. And $\mathbf{n}^{[i]} \in \mathbb{C}^{N}$ are the noise terms for the set of each $i$-th users. $\mathbf{n}^{[i]} \sim \mathcal{C} N\left(0, N_{0} \mathbf{I}_{N}\right)$, i.e., the noise measured at each UT receiving antenna is complex zero mean Additive White Gaussian Noise (AWGN).

\section{1 | Channel model}

The general model [11] for the complex channel matrix is

$$
\mathbf{H}^{[i]}=\mathbf{F}^{[i]} \boldsymbol{\Phi}_{\rho} \mathbf{B}^{[i]} \boldsymbol{\Phi}_{R F}
$$

with $\mathrm{B}^{[i]}$ an $N \times N$ real matrix which models the link budget of each UT, and $\boldsymbol{\Phi}_{p}$ and $\boldsymbol{\Phi}_{R F}$ two diagonal complex $N \times N$ matrices which contain the phase terms arising in the signal propagation and the satellite RF chains, respectively. $\mathrm{F}^{[i]}$ is a diagonal matrix whose non-zero elements, $f_{m m}^{[i]}$, represent a fading coefficient for the $i$-th user in the $m$-th beam. In this paper we will consider Rician fading, with infinite Rice factor for stationary users, and a finite factor for mobile users. The simulation of the mobility is aligned with the importance given by the DVB-S2X standard and some publications, as [12], to the application of precoding to mobile scenarios.

The real matrix $\mathbf{B}^{[i]}$ models each UT link budget and contains the satellite antenna radiation pattern, the path loss and the receive antenna gain. Its $m, n$-th entry is given by:

$$
b_{m n}^{[i]}=\frac{\lambda}{4 \pi d_{m}^{i}} \sqrt{G_{R} G_{m n}^{[i]}}
$$


with $d_{m}^{[i]}$ the distance between the $i$-th UT in beam $m$ and the satellite (slant range), $\lambda$ the wavelength, $G_{R}$ the receiver antenna gain and $G_{m n}^{[i]}$ the multibeam antenna gain between the $i$-th single antenna UT located in the $m$-th beam and the $n$-th on board antenna feed.

The phase of the channel coefficients is divided in two terms. On the one hand, the diagonal matrix $\boldsymbol{\Phi}_{p}$ models the phase originated during the propagation. Here we assume that due to the big slant range the signals of all satellite antennas arrive at the UT with the same phase, this is the reason why this matrix multiplies B by the left. This phase is fixed for each user during all the simulations and it is uniformly distributed between all UTs. Their elements are $\left[\boldsymbol{\Phi}_{p}\right]_{n n}=e^{j \phi_{n}}$, where $\phi_{n}$ is an uniform random variable in $[0,2 \pi)$.

On the other hand, the diagonal matrix $\boldsymbol{\Phi}_{R F}$ models the random phase introduced by the local oscillators, which are independent for each payload chain, and therefore this matrix multiplies $\mathbf{B}$ by the right. The elements of this diagonal matrix are $\left[\boldsymbol{\Phi}_{R F}\right]_{n n}=e^{j \theta_{n}}$, with $\theta_{n} \sim \mathcal{N}\left(0, \sigma_{\theta}^{2}\right)$. A value of $20^{\circ}$ for the variance is used as suggested in [11]. Contrary to $\phi_{n}$, which is fixed during all the simulations, $\theta_{n}$ change with each time realization (transmitted frame).

Lastly, the channel matrix $\mathbf{H}^{[i]}$ for a given time realization is built row by row with the channel vector of the scheduled users, $\mathbf{h}_{k}^{\perp[i]}$. However, the gateway does not have access to the actual channel matrices $\mathbf{H}^{[i]}$ but to the matrices $\hat{\mathbf{H}}^{[i]}$, which include the nullification effect as well as estimation errors. This will be explained in detail in the next section.

\section{2 | Linear precoding}

Let snr $=P_{n} / N_{0}$ be the per beam and per polarization transmit SNR (signal-to-noise ratio), with $P_{n}$ the average per beam and polarization transmit power and $N_{0}$ the noise power at each receiver. The MMSE (Minimum Mean Square Error) precoding matrix is calculated by the gateway by using the average of nullified imperfect CSIT matrices $\hat{\mathrm{H}}^{[i]}$

$$
\hat{\mathrm{H}}_{a v}=\frac{1}{M} \sum_{i=1}^{M} \hat{\mathrm{H}}^{[i]}
$$

Using that matrix, the expression of the MMSE precoder [13] is given by

$$
\mathbf{W}_{\text {mmse }}=\hat{\mathbf{H}}_{a v}^{H}\left(\hat{\mathbf{H}}_{a v} \hat{\mathbf{H}}_{a v}^{H}+\frac{1}{\mathrm{snr}} \mathbf{I}_{N}\right)^{-1}
$$

In order to set the transmitted power, there are several options to normalize (5) such as SPC (Sum Power Constraint), MPC (Maximum Power Constraint) or PLPC (Per Line Power Constraint). SPC, which is used in this paper, can be applied when the satellite payloads have Multiport Amplifiers (MPA) or Flexible TWTAs, that allow the total power $\left(N P_{n}\right)$ to be shared among the different beams. In this case, $\mathbf{W}_{\text {mmse }}$ is multiplied by a scaling factor

$$
\eta=\sqrt{N P_{n} / \text { trace }\left\{\mathbf{W}_{\text {mmse }} \mathbf{W}_{\text {mmse }}^{H}\right\}}
$$

becoming the final precoding matrix

$$
\mathbf{W}=\eta \mathbf{W}_{\mathrm{mmse}} .
$$




\section{3 | Precoded SINRs}

If we refer to the rows of $\mathbf{H}^{[i]}$ and $\hat{\mathbf{H}}^{[i]}$ as $\mathbf{h}_{k}^{[i] \perp}$ and $\hat{\mathbf{h}}_{k}^{[i] \perp}$, respectively, and to the columns of $\mathbf{W}$ as $\mathbf{w}_{k}$ (the precoding vectors), the actual precoded SINR for the $i$-th UT at the $k$-th beam can be calculated as

$$
\operatorname{sinr}_{k}^{[i]}=\frac{\left|\mathbf{h}_{k}^{[i] \perp} \mathbf{w}_{k}\right|^{2}}{\sum_{j \neq k}\left|\mathbf{h}_{k}^{[i] \perp} \mathbf{w}_{j}\right|^{2}+N_{0}}
$$

and the precoded SINR which the gateway estimates for the $i$-th UT at the $k$-th beam, by using the Imperfect CSIT jointly with the precoding matrix is

$$
\sin _{k}^{[i]}=\frac{\left|\hat{\mathbf{h}}_{k}^{[i] \perp} \mathbf{w}_{k}\right|^{2}}{\sum_{j \neq k}\left|\hat{\mathbf{h}}_{k}^{[i] \perp} \mathbf{w}_{j}\right|^{2}+N_{0}} .
$$

We use the notation $\operatorname{sinr}_{k}^{[i]}$ and $\sin _{k}^{[i]}$ to refer to the real and estimated precoded linear SINRs and we reserve the capital letters for their counterpart in logarithmic scale:

$$
\begin{aligned}
& \operatorname{SINR}_{k}^{[i]}=10 \log _{10} \operatorname{sinr}_{k}^{[i]}(d B) \\
& \operatorname{SINRR}_{k}^{[i]}=10 \log _{10} \operatorname{sinr}_{k}^{[i]}(d B) .
\end{aligned}
$$

As we are interested in the SINR errors caused by nullification and their impact on the MCS selection, in the following sections we will characterize the SINR absolute error, defined as

$$
e_{k}^{[i]}=\operatorname{SINR}_{k}^{[i]}-\operatorname{SINR}_{k}^{[i]}(d B)
$$

This is the difference between the SINR estimated by the gateway for each UT (using $\mathbf{W}$ and $\hat{\mathbf{H}}^{[i]}$ ) and the real SINR that the UT undergoes (which depends on $\mathbf{W}$ and the actual channel $\mathrm{H}^{[i]}$ ). If $e_{k}^{[i]}$ is positive -the gateway overestimates the SINR- and no appropriate margins are inserted prior to MCS selection, then transmission errors will take place as a result, even in the absence of other channel imperfections. In the worst case, if the errors last for a large number of frames, re-synchronization might be required.

The selected metric to evaluate the nullification problem is the SINR in $\mathrm{dB}$ absolute error $e_{k}^{[i]}$ as defined in (12). This has the disadvantage of not being normalized and therefore it depends on the transmitted power; in consequence, for higher $P_{n}$ the SINRs and the magnitude of the corresponding SINR absolute errors are higher too. Nevertheless, this metric, contrary to, for example, the relative error, can be translated directly into the margin necessary to subtract from the SINR estimated by the gateway, $\operatorname{SINR}_{k}^{[i]}$, in order to guarantee a robust transmission. To obtain meaningful results with the SINR absolute error, the operation point is selected carefully to reflect a close to reality system.

\section{4 | Scheduling}

In order to emulate a realistic scenario, in the simulations of this article a basic scheduling algorithm is included. However, since the purpose of this article is to evaluate the SINR errors due to the carrier detection errors in the CSI and to propose a solution to overcome the problems they cause, we have maintained the scheduling part as simple as possible. The interested reader can refer to [14], [7] and [15], for example, where the problem of scheduling in 
precoded systems is treated in more detail.

In this article we consider the application of two levels of scheduling: inter-beam and intra-beam. The main goal of the inter-beam scheduling is to allocate simultaneously transmitted frames to groups of UTs which have channels as orthogonal as possible in order to minimize the interference. This scheduling is done heuristically by following a geographical approach, as it was proposed in [15]. The basic idea is to schedule multicast groups in adjacent beams in the same time frame if they are not too close. For achieving this, all the beams are divided into a set of $S$ circular sectors, and we schedule together multicast groups that belong to the same sector in their respective beams [15]. The scheduling algorithm serves sequentially all the sectors in a round robin fashion.

Concerning the inter-beam scheduling, two different scenarios will be tested. On the one hand, a scenario with a random inter-beam scheduling, where the beam is not divided into circular sectors (the whole beam is a sector, $S=1$ ). On the other hand, a scenario where the beams are divided into $S=6$ circular sectors, as depicted in Fig. 1; at a particular time instant, a multicast group is scheduled in the same circular sector for all the beams.

Regarding the intra-beam scheduling, its purpose is to group the users of each circular sector in multicast groups of $M$ users which should meet two prescribed channel conditions. On the one hand, if the users have similar channel vectors $\mathbf{h}_{k}^{[i]}$, the precoding matrix $\mathbf{W}$ built from the average of the channel matrices $\hat{\mathbf{H}}_{a v}$ is better fitted to the users channels. And, on the other hand, if the two users have similar SINRs, the user with the highest SINR does not lose so much capacity due to the use of the same MCS that the user with the worst conditions impose. Inside each circular sector, the available users, i.e., positions from the available radiation pattern, are grouped in groups of $M$ users with the MaxDist algorithm [16] using as features the channel vectors of the UTs and as norm the Euclidean distance.

For the sake of completeness, some details about how the scheduling is implemented to obtain the results of Sections 5, 6 and 7.2 are included here. The multicast groups with all the available positions (UTs) provided by ESA's radiation pattern are created at the beginning of the simulation. And then, each time a sector is served, a different multicast group is selected following a random sequence which covers all groups inside a circular sector. This guarantees that all the users are served fairly and mimics the randomness nature of the traffic, which causes that UTs are scheduled with different users in different beams each time (although always with UTs from homologous sectors for maintaining the inter-beam scheduling policy). In Fig. 1, it can be seen how the served multicast groups, which belong to analogous sectors, are located randomly inside their sectors.

\section{3 | NULLIFICATION DESCRIPTION}

Precoding requires the transmitter to have knowledge of the users channel in the form of CSIT, since this is necessary to compute the precoding matrix $\mathbf{W}$ and estimate the precoded SINR of each user. The CSIT which is available at the gateway is usually imperfect, among other things, due to errors in the estimation of the coefficients of the CSI vectors per the users. However, the main cause of degradation of the CSI is the nullification effect. This is the inability of a receiver to estimate some of the coefficients of the CSI vector due to the low received power of the interfering beams. Thus, a large number of coefficients of the CSI matrix which is used by the gateway are unknown and replaced by zeros. The replacement of these unknown channel coefficients by zeros rather than another arbitrary small value is preferable, so that we avoid guessing about the phase value, which is important to compute the precoding matrix. The channel matrix with this type of degraded CSI, $\hat{\mathrm{H}}$, is said to have nullification errors.

Regarding the synchronization of the carriers transmitted by the different beams, two types of models of satellite systems can be considered, with different consequences on the CSI estimation and nullification. With network synchronization [17], the waveforms received by the user, the desired carrier and the interfering carriers from adjacent 


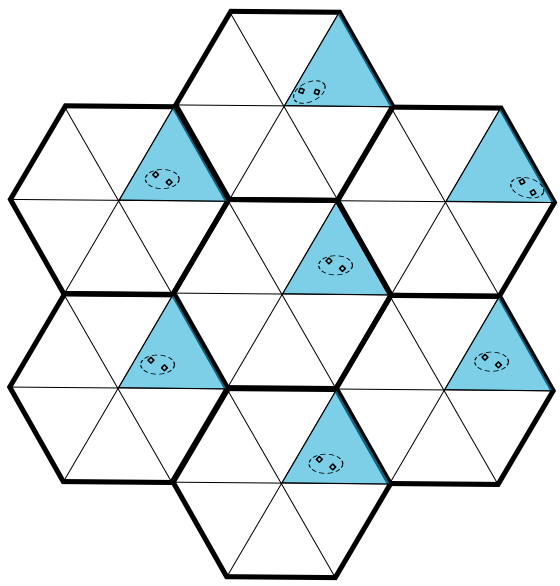

FIGURE 1 Multicast groups served during a transmission interval, showing how the beams are divided into sectors (inter-beam scheduling), and how a random multicast group is served inside each sector (intra-beam scheduling).

beams, can be considered synchronous, i.e. the time delays between the different waveforms is much lower than the symbol period. In the specific case of DVB-S2X, with orthogonal Walsh-Hadamard (WH) sequences for the $P$ pilots, the detection of the interfering carriers is limited mainly by the noise. The $P$ pilots are sequences of known non-precoded symbols which receivers use to estimate the CSI, since the orthogonal WH sequences enable to differentiate up to 31 different interfering carriers. In this situation, the periodical repetition of the 36-pilots blocks along the bundled PLFRAME allows the detection of interfering carriers whose power is even $15 \mathrm{~dB}$ below the noise. If pilots across more than one superframe are averaged, this threshold can be further reduced. When the carriers are not synchronized, due to, among other factors, different delays in the payload circuits, the orthogonality of the $\mathrm{WH}$ sequences can not be exploited, and the estimation becomes limited fundamentally by the interference [18]. In this asynchronous system, interfering carriers can be estimated even when they are approximately up to $15 \mathrm{~dB}$ below the desired carrier. Recent research showed that for leveraging all the potential of precoding in multibeam satellite systems the carriers should be synchronized to a great extent [17]. Moreover, if the different carriers transmitted by the satellite are substantially synchronized, the computational burden for obtaining the CSI at the receiver side can be reduced [19].

Mathematically, for simulating the nullification effect under purely asynchronous or synchronous carriers, we construct the nullified matrix $\hat{\mathrm{H}}$ in two different forms, depending on the system type [1]:

1. Asynchronous system: Since our assumption is to work in clear sky conditions, the precoded $\mathrm{C} / \mathrm{N}$ is relatively high so that the nullification in the asynchronous system is driven mainly by the interference power more than the noise power. Therefore, we assume that the nullification is performed based on a I/C threshold $T_{h}$ :

$$
\hat{h}_{i j}= \begin{cases}0 & \text { if } 20 \log _{10}\left|h_{i j} / h_{i i}\right|<T_{h} \\ h_{i j}, & \text { otherwise. }\end{cases}
$$


2. Synchronous system: the nullification is performed based on a $\mathrm{I} / \mathrm{N}$ threshold $T_{h}$ :

$$
\hat{h}_{i j}= \begin{cases}0 & \text { if } 20 \log _{10} \sqrt{\mathrm{snr}}\left|h_{i j}\right|<T_{h} \\ h_{i j}, & \text { otherwise. }\end{cases}
$$

The interested reader can find in [1] a comparison of the SINR absolute errors with different thresholds for synchronous and asynchronous nullification. However, the different beam carriers are not fully asynchronous or synchronous in practice, and the capability of a practical receiver to estimate a given interfering carrier depends on both $\mathrm{C} / \mathrm{N}$ and $\mathrm{C} / \mathrm{l}$. Fig. 3 shows the $(\mathrm{C} / \mathrm{N}, \mathrm{C} / \mathrm{I})$ regions for which estimation is possible and for which nullification takes place. This performance of the CSI estimation is achieved by using the architecture shown in Fig. 2 and is referred hereafter as real nullification. From that figure it can be inferred that this realistic nullification is similar to a synchronous nullification with an $\mathrm{I} / \mathrm{N}$ threshold $T_{h}=-12 \mathrm{~dB}$.

The only reference the authors are aware of regarding the required thresholds for CSI estimation is the DVBS2X implementation guidelines [20]. Therein, performance results of several precoding systems are presented under different levels of nullification, provided in terms of a threshold in the $\mathrm{C} /(\mathrm{N}+\mathrm{I})$ of the $\mathrm{CSI}$ coefficients. Thresholds ranging from $-12 \mathrm{~dB}$ to $-21 \mathrm{~dB}$ are considered for simulating an imperfect CSI. If we look at Fig. 2 for the lowest $\mathrm{C} /(\mathrm{N}+\mathrm{l})$ for which a channel coefficient can be estimated, in what we call real nullification (it happens at the lower left corner of the green area), we observe that the SINR value is $-10.4 \mathrm{~dB}$. Therefore, our realistic assumptions consider a CSI even more imperfect than the assumptions of [20].

One of the most challenging and delicate phase for a precoded system to fully exploits its advantages is the proper and accurate recovery of the CSI for the main (reference) and the interfering carriers [19]. The implementation guidelines [20] of the DVB-S2X standard propose a method to compute the CSI which requires that each terminal must lock on each waveform (the reference signal intended for the user and the interfering signals from the other beams), since the different waveforms transmitted by the satellite are not considered synchronous. Clearly, such an architecture entails a high computational complexity since it requires to apply independently a frame, time, frequency and phase synchronization for each carrier. This set of repeated synchronization chains can indeed be highly demanding for a commercial receiver.

The recent patent [19] proposes a solution to overcome the computational complexity problem of the CSI acquisition in multibeam precoding systems. In this patent, it is assumed that the different carriers transmitted by the satellite are synchronized in time and frequency with respect to each other. This can be accomplished by means of a common Ultra Stable Oscillator, which provides the reference clock for all the down-conversion chains, and also by applying some calibration procedures through dedicated terminals, see for example [17]. Fig. 2 shows an UT architecture for calculating the CSI similarly to that proposed in the patent. Since the different waveforms are synchronized in both time and frequency, synchronization procedure is performed only once against the reference waveform, reducing the complexity and allowing the estimation of more CSI coefficients by a practical receiver.

As stated above, the CSI estimation performance in Fig. 3, which will be used later to study the SINR errors due to nullification, corresponds to the architecture in Fig. 2, which assumes a synchronous system. After the coarse frequency recovery, a frame synchronization is performed based on the signature (WH sequence) of the reference carrier, while, for each interfering carrier, a verification stage based on carrier specific signatures is carried out, as can be seen in Fig. 2. The architecture assumes each carrier to be synchronous in frequency while not in phase and, as a consequence, it performs a frequency/phase noise tracking on the main carrier (reference waveform) and use the same tracking values to compensate the interfering waveforms.

Using this approach, the CSI estimation stage provides a constant (during the channel coherence period) phase 
value normalized to the main signal phase variation. In fact, while the phase of the superimposed signal is affected not only by the phases of the relative channel vector but also from the receiver impairments (LNB frequency error, phase offset), what the terminal is practical able to estimate is the interfering waveforms phase differences from the tracking phase values of the main carrier, which should be almost constant (regardless of phase noise differences amongst transponders) within a certain coherence window.

In practice, the interfering carriers which are detected suffer also from estimation errors. Those carriers in the shaded area in Fig. 3 are estimated with an error which will be a function of both the $\mathrm{C} / \mathrm{N}$ of the user and the $\mathrm{C} / \mathrm{I}$ of the particular CSI coefficient. This error, modeled as Gaussian, will be higher for those carriers near the miss-detection boundary. The simulations included later in the paper will account for these random estimation errors:

$$
\hat{h}_{i j}=h_{i j}+e_{i j}
$$

with $e_{i j}$ following a Gaussian distribution with parameters detailed in Tab. 1. The mean and standard deviation of the phase estimation error are given in absolute terms, whereas they are given in relative terms for the amplitude, to be independent of the channel coefficients magnitude. In both cases, maximum and mode are shown.

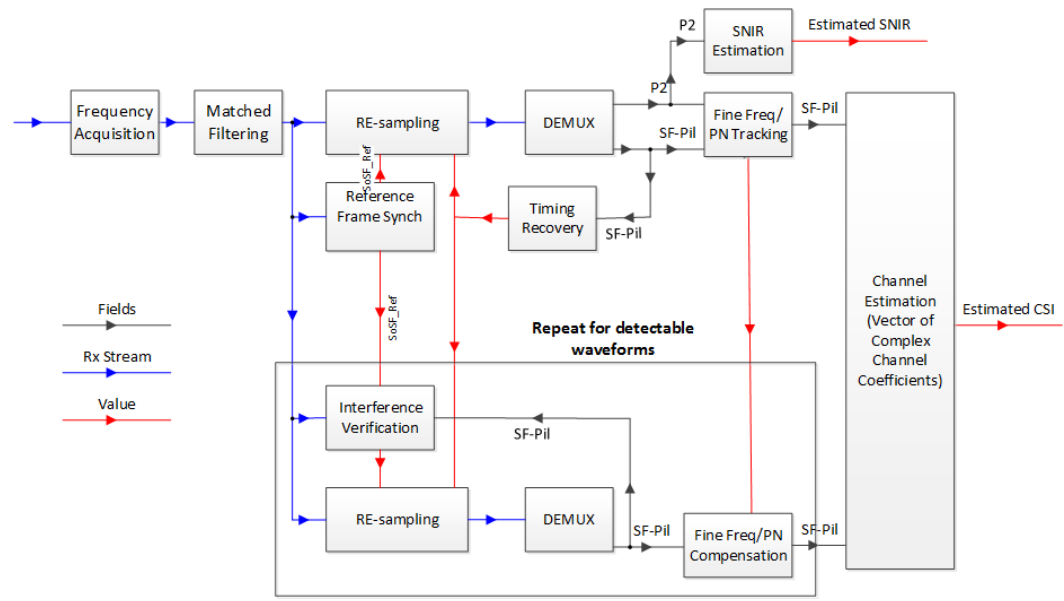

FIGURE 2 Architecture of the receiver for CSI detection and estimation.

\section{4 | SYSTEM PARAMETERS}

The main parameters of the Ka-band multibeam satellite system used in the simulations are collected in Table 2. The satellite covers most part of the continental Europe as can be seen in Fig. 4, which shows the beams boundaries over the map of Europe. A 245 beams multibeam channel, generated based on ESA's radiation pattern [6], is used as basis for the simulations. For each beam a grid with discrete positions is available, with the number of positions of the beams ranging from 68 to 108 . With the parameters from Table 1, the clear sky non-precoded $\mathrm{C} / \mathrm{N}$ of the users ranges from 7 to $13.75 \mathrm{~dB}$, as can be seen in the histograms of Figs. 5-7.

Three different system configurations are used in the simulations. One system with all the 245 beams (see Fig. 5) and two small size systems which consist of a cluster of only 9 beams, one located in the center of Europe (Fig. 


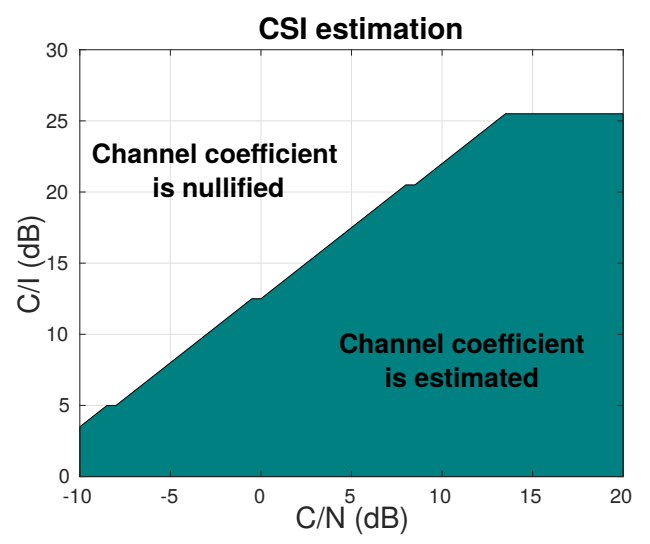

FIGURE 3 Range of values of $\mathrm{C} / \mathrm{N}$ and $\mathrm{C} / \mathrm{I}$ which allow carriers estimation in real nullification.

Error amplitude $\left|e_{i j}\right|$

\begin{tabular}{l|l|l} 
Mean relative error & Maximum: 0.17 & Mode: 0.05 \\
\hline Relative standard deviation & Maximum: 0.02 & Mode: 0.005
\end{tabular}

Error phase $\angle e_{i j}\left({ }^{\circ}\right)$

\begin{tabular}{l|l|l} 
Mean error & Maximum: $3^{\circ}$ & Mode: $0^{\circ}$ \\
\hline Standard deviation & Maximum: $1.2^{\circ}$ & Mode: $0.7^{\circ}$ \\
\hline
\end{tabular}

TABLE 1 Characterization of the CSI estimation errors.

6) and other located at the edge of the coverage, in the South West of the lberian Peninsula (Fig. 7). Note that the bandwidth and power parameters in Table 2 correspond to carriers of 10 Mbaud and roll-off of 0.2.

The size of the multicast groups $M$ deserves a special comment. It is a well-known fact in the literature, [21], [22], that the performance of precoded systems degrades rapidly with the number of mulicast users per frame $M$. For this reason recent studies from ESA [10] propose some modifications to the DVB-S2X waveform in order to have a better performance with precoding. They propose to use shorter frames of 2048 (2K), 4096 (4K) and 8192 (8K) symbols, with a constant frame length on air, not depending on the selected MODCOD, and new FEC schemes allowing for variable FEC frame length.

These short frame lengths justify the use of a small number of multicast users $M$ per frame. Assuming long packets of length 1518 bytes (12144 bits), corresponding to the Ethernet Maximum Transmission Unit (MTU), and a maximum efficiency of $5 \mathrm{bits} / \mathrm{s} / \mathrm{Hz}$, in $2 \mathrm{~K}, 4 \mathrm{~K}$ and $8 \mathrm{~K}$ frame lengths there is room for up to $0.84,1.68$ and 3.37 packets, respectively. The number of multicast users that can be scheduled on these shorter frames depends on several factors, as can be the proper frame length, the MODCOD or spectral efficiency of the frame and the size of the user packets. An intelligent selection of these parameters by the scheduler, taking also into account other factors such as the latency, can help to reduce the number of multicast users per frame, thus avoiding a performance degradation. For example, 


\begin{tabular}{|l|c|}
\hline Parameter & Value \\
\hline Satellite orbit & GEO \\
\hline Downlink frequency & Ka-band $(20 \mathrm{GHz})$ \\
\hline Number of beams $N$ & 245 or 9 \\
\hline Size of multicast groups $M$ & 2,3 and 4 \\
\hline \# of sectors per beam $S$ (inter-beam sch.) & 1,4 or 6 \\
\hline Receive antenna gain & $40 \mathrm{dBi}$ \\
\hline Noise temperature $T$ & $235.3 \mathrm{~K}$ \\
\hline Receiver G/T & $16.3 \mathrm{~dB} / \mathrm{K}$ \\
\hline Noise bandwidth (roll-off included) & $12 \mathrm{MHz}$ \\
\hline Per beam and polarization transmit power $P_{n}$ & $0.15 \mathrm{~W}(21.76 \mathrm{dBm})$ \\
\hline Total power per carrier & $36.75 \mathrm{~W}(15.65 \mathrm{dBW})$ \\
\hline
\end{tabular}

TABLE 2 System parameters.

the selection of a short frame length of $2 \mathrm{~K}$ symbols, jointly with a low spectral efficiency, can help to limit the number multicast users per frame when small packets are scheduled. Another alternative could be the scheduling of a large packet jointly with a pair of small packets in the same frame, in order to maintain a low number of multicast users $M$ per frame. Having all these ideas in mind, in this article, simulation results are provided mainly for $M=2$. Despite of this, we have included some statistics of the SINR absolute error also for $M=3$ and $M=4$.

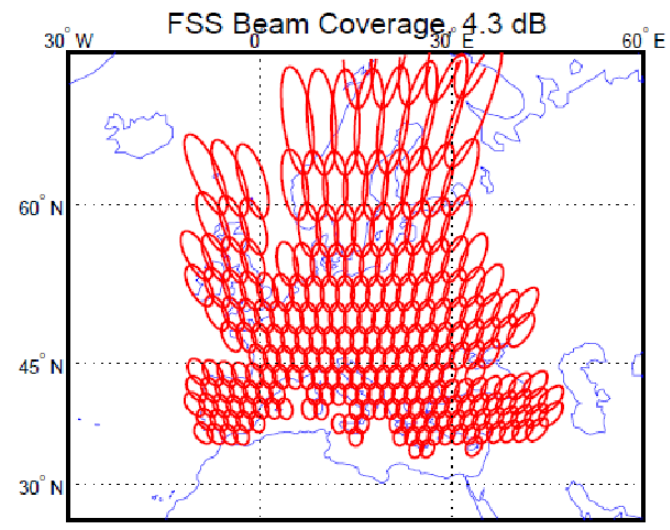

FIG URE 4 Map of the satellite coverage over Europe with the beams boundaries.

Fig. 8 contains a map with the number of estimated carriers per position across the satellite coverage using the real nullification model explained in Section 3 (estimation performance of Fig. 3 and Tab. 1) and for the system parameters of Tab. 2. It can be seen that the number of estimated carriers ranges between 1 and 15 . In the edge of the satellite coverage typically less than 6 carriers are estimated and far from the edges this number increases, 


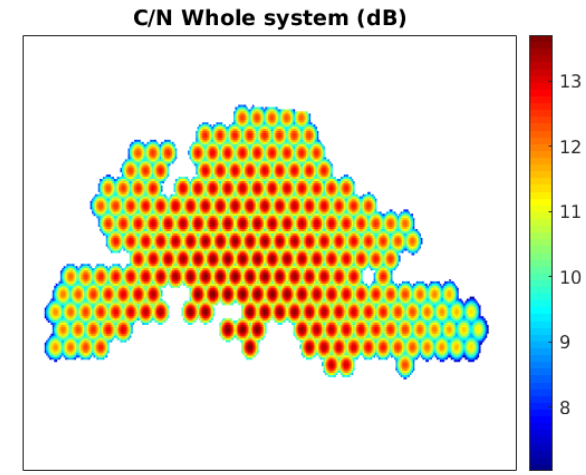

(a) Map of $\mathrm{C} / \mathrm{N}(\mathrm{dB})$.

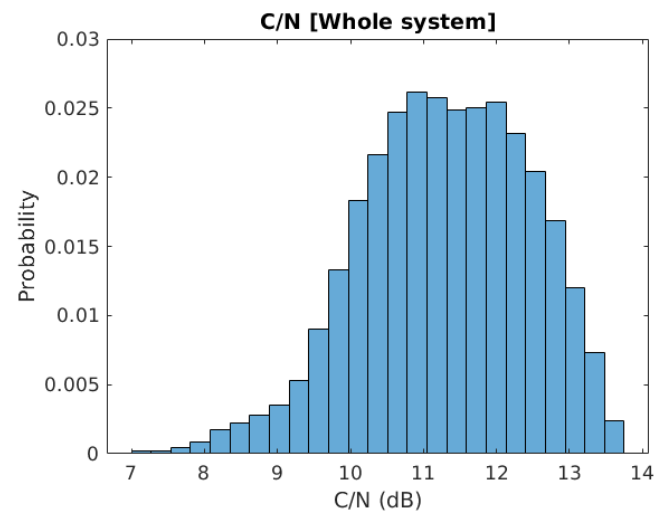

(b) Histogram of the $\mathrm{C} / \mathrm{N}$ distribution.

FIGURE 5 Map of the C/N per position in the satellite coverage and histogram with its distribution for the system with all the 245 beams.

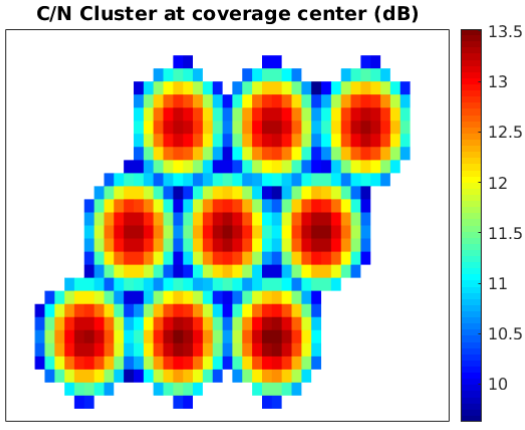

(a) Map of $\mathrm{C} / \mathrm{N}(\mathrm{dB})$

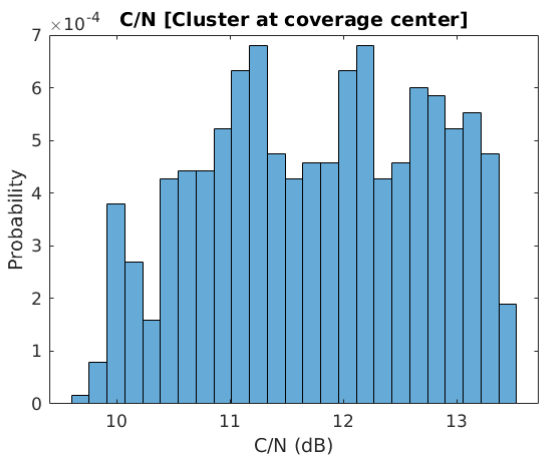

(b) Histogram of the $\mathrm{C} / \mathrm{N}$ distribution.

FIGURE 6 Map of the C/N per position and histogram with its distribution for the cluster of 9 beams located at the center of Europe.

lying between 10 and 15 . This last number is the half of the maximum number of carriers which can be differentiated with the WH sequences (32) and much lower than the number of total beams, 245. This map demonstrates that the nullification must be taken into account when precoded systems are analyzed, since it is an important source of CSI degradation.

\section{5 | SINR ERRORS DUE TO THE NULLIFICATION}

In this section simulation results of the SINR absolute error due to nullification and CSI estimation errors will be provided from three different perspectives. Firstly, Subsection 5.1 presents several statistics of the aggregated SINR 
$\mathrm{C} / \mathrm{N}$ Cluster at coverage edge (dB)

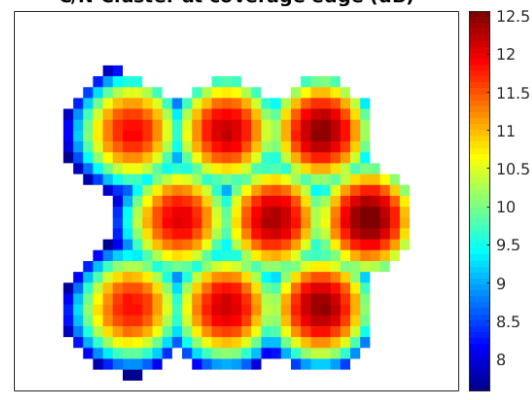

(a) Map of $\mathrm{C} / \mathrm{N}(\mathrm{dB})$

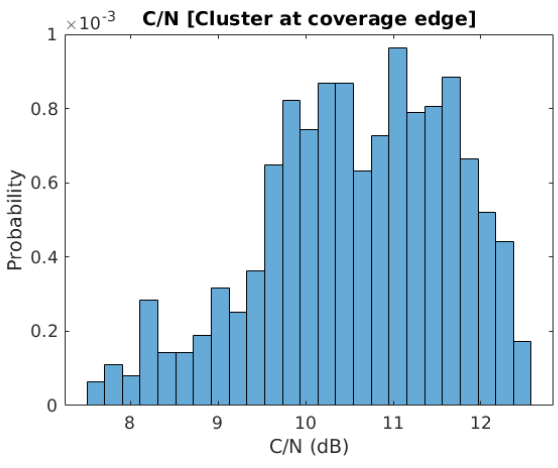

(b) Histogram of the $\mathrm{C} / \mathrm{N}$ distribution.

FIGURE 7 Map of the $\mathrm{C} / \mathrm{N}$ per position and histogram with its distribution for the cluster of 9 beams located at the South West of the Iberian Peninsula

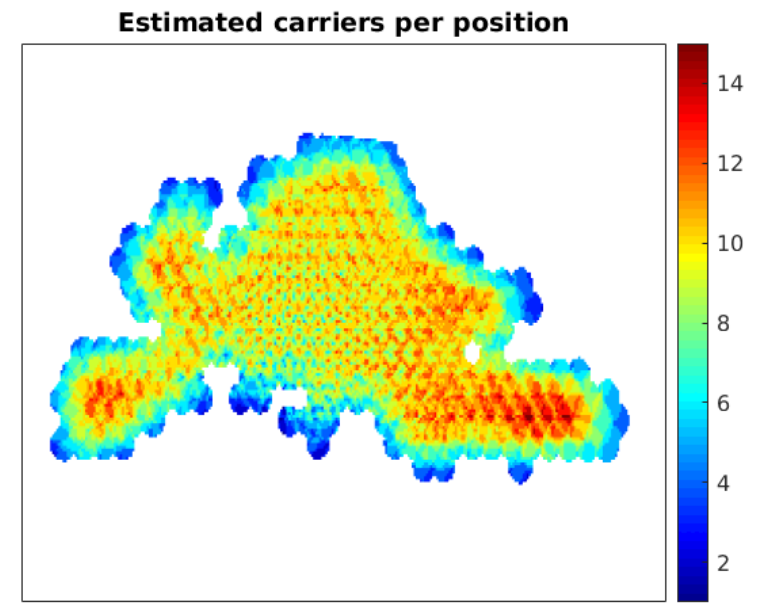

FIGURE 8 Map of the satellite coverage with the number of coefficients of the CSI vector estimated per position when using real nullification.

error, testing all user locations on the satellite coverage and showing the global results. Then, Subsection 5.2 analyses the SINR absolute error adding the spatial dimension of the data in order to reveal the geographical dependence of the error. Lastly, Subsection 5.3 focus the attention on the statistics of the SINR and the SINR absolute error from the point of view of specific users, i.e., at a fixed location.

Each simulation comprises the transmission of 1,000 frames. Previously, multicast groups with $M=2$ users are created in each circular sector of each beam by using the scheduling algorithms detailed in Section 2.4 and considering as active all the positions of the beam. In some cases, multicast groups of size $M=3$ and $M=4$ are also considered. Then, once selected the $2 N$ users scheduled in each frame, the channel matrices $\mathrm{H}^{[i]}$ are built with the channel vectors 
of the users, the imperfect CSIT with the nullification and estimation errors $\hat{\mathrm{H}}^{[i]}$ is obtained and the precoding matrix $\mathbf{W}_{m m s e}$ is calculated using the average matrix channel matrix $\hat{\mathbf{H}}_{a v} \cdot \operatorname{In}$ the simulations of this section the Rician fading is not included. $f_{m m}=1$ in the matrix $\mathrm{F}^{[i]}$ of the channel model, equation (2). Afterwards, the actual UT SINRs SINR ${ }_{k}^{[i]}$ and the corresponding estimated value by the gateway, $\operatorname{SINR}_{k}^{[i]}$, are obtained. Lastly, its difference gives the SINR absolute error $e_{k}^{[i]}$, which is analysed from three different perspectives along the following subsections.

\section{1 | Aggregated results}

In this subsection a collection of results of the SINR absolute error, as defined in Equation (12), is presented. Firstly, the bar diagrams of Fig. 9a show the maximum SINR absolute error by using the simple model for synchronous nullification of Equation (14). Results are shown for the system with 245 beams, with 6 circular sectors for the interbeam scheduling, multicast groups of size $M=2$, and for two different values of the transmitted power $P_{n}$ : the nominal operation point of Table 2 , and a second power value which is $3 \mathrm{~dB}$ lower. Three different $\mathrm{I} / \mathrm{N}$ nullification thresholds are studied in order to link the performance in the interfering carriers detection with the maximum SINR error obtained. Moreover, results for the realistic nullification depicted in Fig. 3 are included for comparison. In the light of data from Fig. 9a, for the maximum SINR error being lower than $1 \mathrm{~dB}$ an $\mathrm{I} / \mathrm{N}$ close to $-20 \mathrm{~dB}$ is needed in synchronous systems. This graphic illustrates that, if CSI detection is improved by reducing the I/N detection threshold (this can be achieved by averaging the pilot symbols during longer periods), the ensuing SINR error due to the nullification will be lower. Lastly, it can be seen how the maximum SINR absolute error depends also on the operation point. If the power is lower, the SINR will be lower too and the magnitude of the errors will be also reduced.

Now, Fig. 9b compares the maximum SINR absolute error when real nullification is used, i.e., applying both $\mathrm{C} / \mathrm{N}$ and $\mathrm{C} / \mathrm{I}$ thresholds of Fig. 3 to decide if a coefficient of the channel vector is nullified. We show results with the nominal power of Tab. 2 for three different systems: one with all the satellite beams $(N=245)$, one consisting of a cluster with only $N=9$ beams located at the center of Europe, and another with a cluster of also $N=9$ beams but at the edge of the coverage. We also compare two scenarios, one without inter-beam, i.e. $S=1$ sectors, (blue bars) and another with a inter-beam scheduling algorithm which creates $S=6$ circular sectors in the beams.

The maximum error for the complete system using inter-beam scheduling is $1.6 \mathrm{~dB}$, which is reduced to 1.4 and 1.0 $\mathrm{dB}$ for the systems with a reduced set of beams. The reason why the cluster at the edge has a lower error is because in that location the $\mathrm{C} / \mathrm{N}$ is smaller compared with the other two scenarios, as it can observed in the histograms of Figs. 5-7. Regarding the error in the 245-beams system, it is bigger than in the reduced-size clusters because the proportion of nullified coefficients in the channel vector of $N$ is much higher. The accumulation of many small differences among $\mathbf{h}_{k}^{[i] \perp} \mathbf{w}_{j}$ and $\hat{\mathbf{h}}_{k}^{[i] \perp} \mathbf{w}_{j}$ when calculating the numerator and denominator of the SINR in the Equations (8) and (9) causes the error to be bigger in the complete system. Lastly, when comparing the blue and red columns we observe that the absence of a proper inter-beam scheduling algorithm increases slightly the error.

The plot of Fig. 9c shows, for the 245-beams system with 6 sectors in the inter-beam scheduling, the experimental Complementary Cumulative Distribution Function (CCDF) of the SINR error with the real nullification. The CCDF provides the probability of the SINR error being larger than a given abscissa value and offers some insight into the value of the margin required for guaranteeing a given target Frame Error Rate (FER) across the satellite footprint. Frame refers to the physical layer unit which contains a codeword with data for $M$ users encoded using the same MCS. For example, from this plot it can be stated that the subtraction of a $1.3 \mathrm{~dB}$ margin from the estimated SINR by the gateway should help to guarantee a FER of $10^{-4}$ for the nominal transmit power case.

For the system with 245 beams, real nullification and the nominal power, we have obtained statistics of the SINR absolute error for different values of the multicast group size $M$. In Fig. $9 \mathrm{~d}$ the 0.99999 -quantile of the SINR absolute 
error distribution is shown by means of vertical bars for $M=2, M=3$ and $M=4$, comparing also the two scheduling options: $S=1$ sectors (no inter-beam scheduling) and $S=6$ sectors in the inter-beam scheduling. This value gives an idea of the global margin required for a FER of $10^{-5}$. It can be seen that the error is reduced when $M$ grows, fact that could be caused by the averaging of more channel matrices for computing the precoding matrix.

These results show that even in the absence of other imperfections, nullification itself can degrade the performance of multibeam satellite systems which use precoding unless proper countermeasures are applied. An error larger than $0 \mathrm{~dB}$ means that the gateway is overestimating the actual UT SINR, and it implies that if $\operatorname{SINR}_{k}^{[i]}$ is used directly to allocate an MCS to the UT at beam $k$, an error in the transmission will occur whenever the error is bigger than the distance of $\operatorname{SINR}_{k}^{[i]}$ to the threshold SINR of the MCS used in the transmitted frame. And since the spacing between two DVB-S2X consecutive MCS is typically between 0.3 and $1.0 \mathrm{~dB}$, and as the SINR errors exceed these values with a non-negligible probability, the FER will be higher than the QEF target of $10^{-5}$, one erroneous frame out of 100,000 .

\section{2 | Spatial analysis}

In order to detect a potential geographical dependence of the SINR absolute error $e_{k}^{[i]}$, we have used some of the simulation data employed to plot the results of Fig. 9 to create the maps of Fig. 10. The map in Fig. 10b shows the maximum error per position after the transmission of 1, 000 frames in the complete system ( 245 beams), with the nominal power $P_{n}$ of Tab. 2, $S=6$ sectors for the inter-beam scheduling and multicast group of size $M=2$. Although there are some hot points where the error reaches values as high as $1.5 \mathrm{~dB}$, the vast majority of the positions have a lower error slightly higher than $0 \mathrm{~dB}$ in the coverage center and even lower than $0 \mathrm{~dB}$ in some edge areas. The difference between the lowest and highest maximum error per position is as big as $2.5 \mathrm{~dB}$. Then, the map of Fig. 10a sums up the information of the previous map showing the maximum error in each beam, aggregating all the beam positions. Again, the same trend is observed: beams in the center of the coverage tend to have higher errors and the maximum SINR absolute error is lower at the edge of the coverage. The higher $\mathrm{C} / \mathrm{N}$ in the central beams (as can be seen in the map of Fig. 5a), together with the large number of beams in their vicinity, increases the error at those central beams.

A rough countermeasure for the errors caused by the nullification could be the subtraction of a global margin to the SINRs estimated by the gateway. This margin could be obtained from the CCDF of the SINR absolute error (shown in Fig. 9c), by using the target FER. However, the results of the geographical distribution of the error show that a global margin to be applied throughout the whole footprint can be quite inefficient, since the error magnitude differs significantly across the footprint. Furthermore, remarkable differences appear within the same beam from one position to another. As a result, a per user margin seems a better solution.

\section{3 | Statistical analysis at fixed locations}

This subsection deals with the characterization of the SINR and the associated SINR error in a fixed position. If a pair of users is always scheduled together with the same set of users in all the other $N-1$ beams, the SINR and the SINR absolute error will be constant with time in the absence of other imperfections such as channel fading, phase noise or Gaussian estimation errors in the CSI. However, the user grouping is not kept fixed with time, due to the random nature of the traffic, among other reasons.

Focusing on a specific user, Fig. 11 shows the time evolution of the actual SINR, SINR ${ }_{k}^{[i]}$, and the associated error, $e_{k}^{[i]}$, along with their respective empirical probability density functions. The dashed lines of the upper-left represent the threshold SINRs of the DVB-S2X MCS. It can be seen that both SINR and SINR error tend to have a Gaussian 


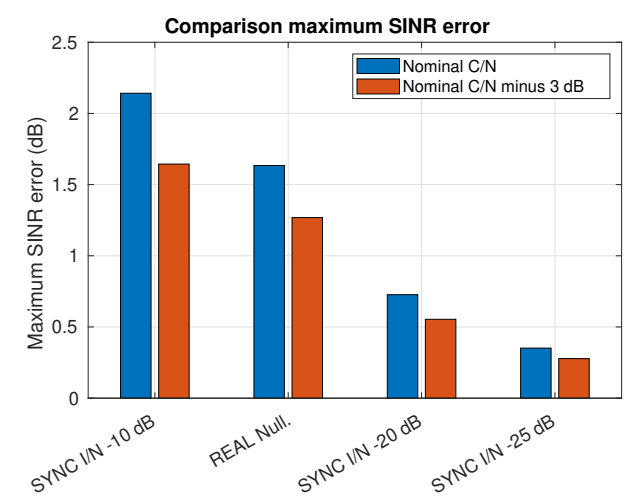

(a) Effect of operation point $(\mathrm{C} / \mathrm{N})$ and detection performance (245-beams system, synchronous nullification, $M=2$ ).

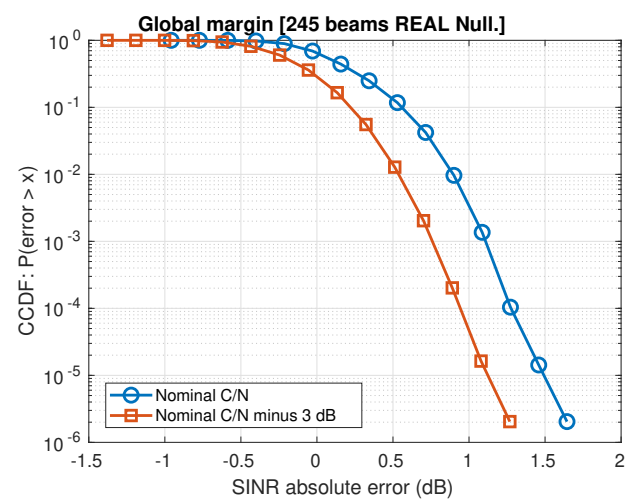

(c) SINR error CCDF with real nullification (245-beam system, $S=6$ sectors and $M=2$ ).

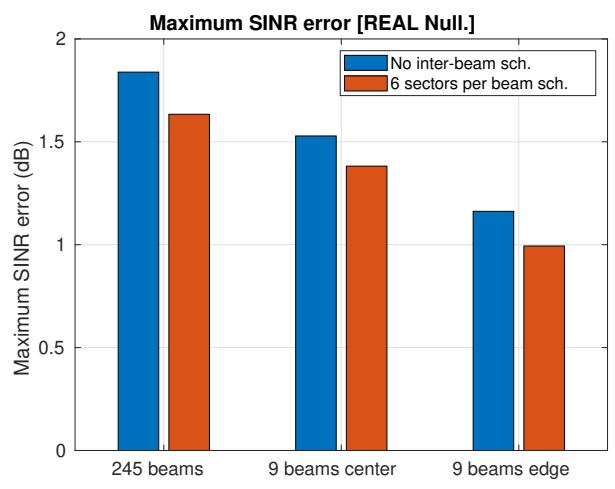

(b) Effect of inter-beam scheduling and system size (real nullification with $M=2$ ).

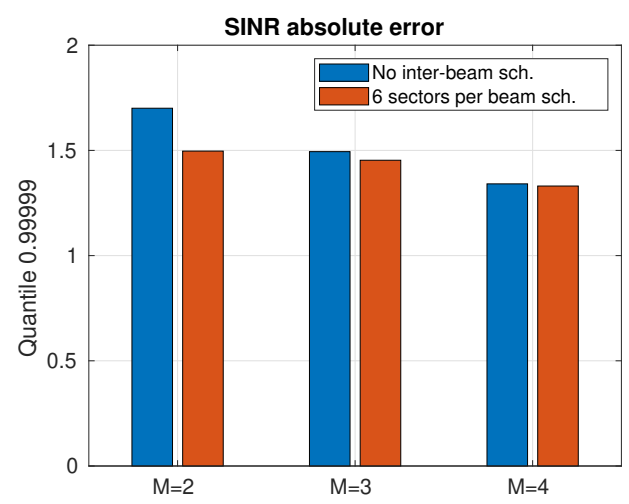

(d) SINR error 0.99999-quantile for different multicast group size $M$ (245-beam system, real nullification)

FIGURE 9 Maximum SINR absolute error over 1,000 realizations in (a) and (b), CCDF of the SINR absolute error with real nullification in (c) and 0.99999-quantile for different multicast group size $M$.

distribution. Fig. 11 is used as illustration, since similar plots were obtained in all the examined positions for three different scenarios: without fading, Rician fading with Rice factor $K=25 \mathrm{~dB}$, and Rician fading with Rice factor $K=34$ $\mathrm{dB}$. Furthermore, we observe that both SINR and SINR error show a stationary behavior, with their respective mean and variances remaining quite stable with time.

\section{6 | SYSTEM THROUGHPUT AND BACK-OFF MARGIN}

In the previous section several statistics of the SINR absolute error caused by the nullification have been shown. Although the maximum SINR error in the whole footprint can reach high values (take, for example, $1.6 \mathrm{~dB}$ with realistic nullification, nominal transmit power and 6 sectors per beam in the inter-beam scheduling), in general, the error is much 


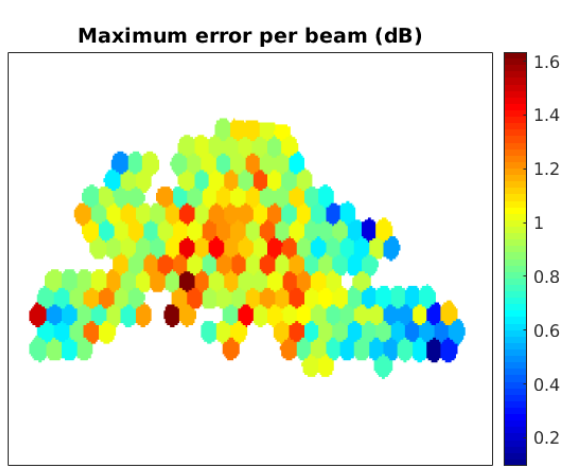

(a) Maximum error per beam.

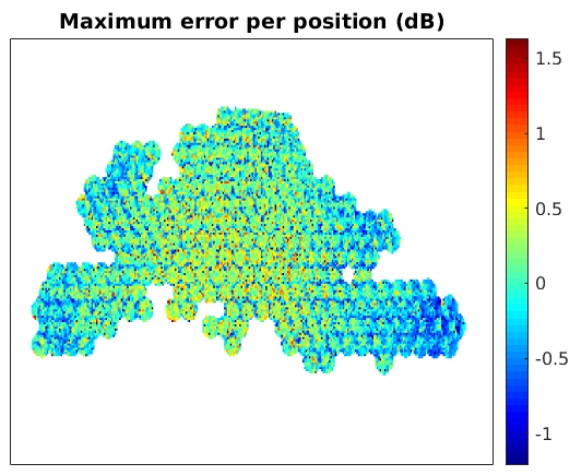

(b) Maximum error per position.

FIGURE 10 Maps of the SINR maximum error with real nullification for the complete system, nominal power, $S=6$ sectors per beam and $M=2$.
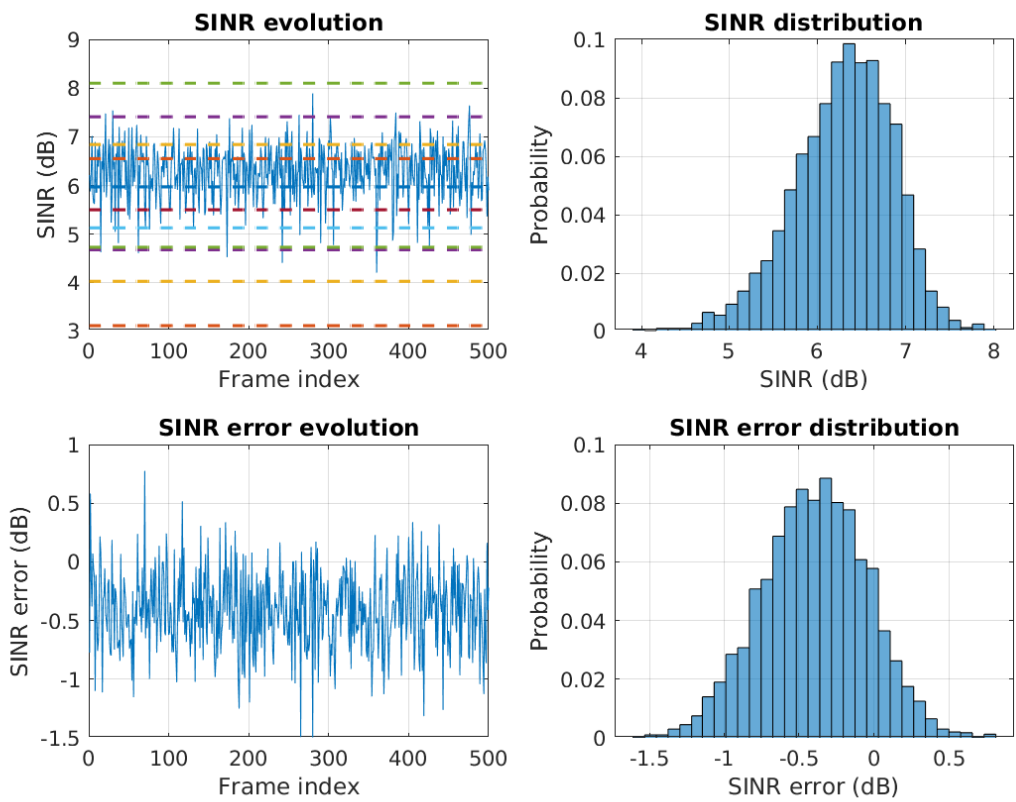

FIGURE 11 Example of SINR and SINR error time evolution and their distribution, at a fixed location.

lower in the vast majority of the positions, as can be seen in Fig. 10b.

When the SINR absolute error of an user is negative, it means that the gateway is underestimating its actual SINR. Therefore, it will allocate a spectrally underperforming MCS, leading to an under-utilization of the available resources. 
On the other hand, when the SINR absolute error of an user is positive, the gateway is overestimating its actual SINR. This case is even more critical than the previous one, because the MCS allocated to the frames of the user is very likely beyond its decoding capabilities, leading to an erroneous transmission which increments the FER and implies a waste of resources.

One possible solution to avoid the errors caused by the SINR overestimation consists on applying a safety or back-off margin to the users' SINR estimated by the gateway. The subtraction of a positive margin from the SINR when choosing the MCS of each transmission helps to maintain the FER around a predefined target level. Depending on the granularity of this margin three options can be taken: a unique global margin for all the satellite coverage, a beam-dependent margin, and a user-dependent margin.

In this section we want to find out the throughput degradation due to imperfect and nullified CSIT depending on the type of margin used with respect to a system with perfect CSIT. Fig. 12 compares the achieved throughput with perfect CSIT and imperfect CSIT due to the nullification; 6, 4 and 1 circular sectors per beam are considered for the inter-beam scheduling, and the three types of the aforementioned margins are evaluated. For each simulation the average throughput per frame is calculated after 10,000 realizations, by using the spectral efficiency of DVB-S2X MCS, shown in Fig. 13, and grouping $M=2$ users per frame. Then, the throughput of each simulation is normalized after dividing it by the throughput of the perfect CSIT case with 6 sectors. It can be readily seen that the use of a proper inter-beam scheduling algorithm with 4 or 6 circular sectors per beam increases the throughput. It can be also observed the strong impact on the throughput of the type of margin. More specifically, for 6 sectors per beam, if we use a global margin by subtracting the maximum error observed in the system from all the SINRs calculated by the gateway before selecting the MCS, the throughput falls $21 \%$ as compared with the perfect CSIT scenario (see purple bar). Instead, if a different margin per beam is applied, the throughput loss is reduced to $16 \%$ (see yellow bar). Fig. 10a displays differences in the maximum error per beam of about $1.4 \mathrm{~dB}$, giving credit to the benefits when increasing the granularity of the margin. Lastly, the throughput when an independent per user margin is employed is shown with orange bars in Fig. 12. In this case, the throughput is obtained after subtracting from each user SINR the maximum SINR error that is observed in its position. As Fig. 10b anticipates, the use of an individual margin per UT is clearly the best option to compensate for the errors that the nullification causes in the SINR estimation. By employing a user-dependent margin, only a $6 \%$ of throughput is lost when compared with the perfect CSIT scenario.

As discussed, nullification is a major impairment affecting the estimation of the SINR at the transmitter. However, additional sources of imperfection affecting also the quality of the available CSIT take place in practice such as the outdated estimation of the SINR, due, for example, to rapid channel variations, at least faster than the tracking ability of the transmit-receive loop. Due to this and other practical reasons, the user-dependent margin should be adaptive, so that the rate of erroneous frames can be properly bounded. We detail in the next section how this margin can be conveniently adapted; a multibeam satellite system with ACM and precoding, severely affected by nullification and Rician fading, will be simulated for illustration purposes.

\section{7 | LINK ADAPTATION ALGORITHM}

In this section we introduce an enhanced link adaptation algorithm taken from [9], which is based on the use of an adaptive margin as a countermeasure for the errors that nullification and other CSI imperfections can cause. The algorithm performance will be evaluated through simulations. The aim of the algorithm is to select the MCS of each frame for each UT, maintaining the FER around a target value $p_{0}$. The link adaptation procedure must be performed at the gateway side rather than at the UTs, since the gateway is the only entity with all the required information to 


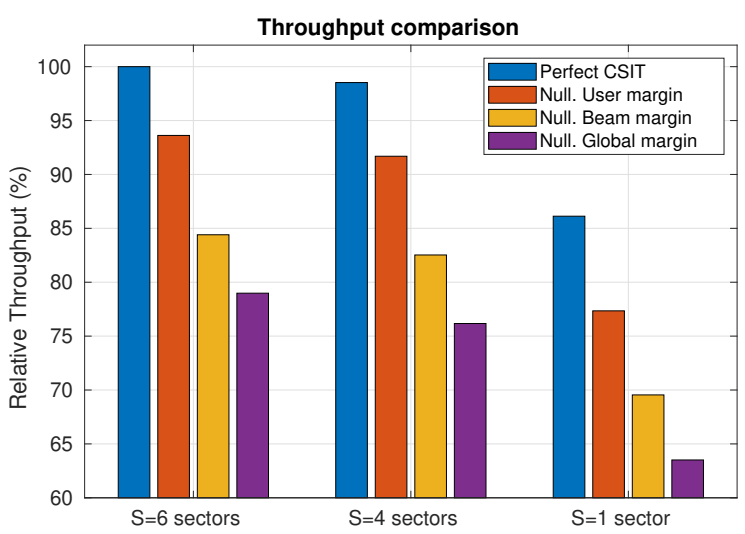

FIGURE 12 Throughput comparison of a 245 beams system (real nullification, nominal power and $M=2$ ) with perfect and nullified CSIT depending on the type of margin used and the number of circular sectors in inter-beam scheduling.

calculate the SINRs, namely, the channel vector of all scheduled users and the precoding matrix employed in each frame. Due to all the considerations explained previously, the gateway will run an independent margin per UT.

In the link adaptation algorithm described in [9] with terrestrial systems in mind, individual ACKs and NAKs are sent for each transmitted frame. However, when retransmissions are not required, as it is the case of DVB-S2(X) links which operate at a very low FER, this algorithm can work also with statistical information about the errors. Thus, the overload of the return link is also avoided, since it suffices to inform about the number of erroneous frames after the reception of a given number of $N_{f r}$ frames. This encoding of the error statistics requires the transmission of a very small amount of bits as compared with the amount of CSI feedback in a precoded system. According to the DVB-S2X standard [2], up to 1024 bits could be required every 500 ms to inform about the CSI. In this paper we have employed individual acknowledgements of the frames to ease the ACM simulations, but the reader must bear in mind that for high frequency broadband DVB-S2X networks, the use of statistical information about the link errors is preferable.

\section{1 | Algorithm description}

The adaptation of the margins using the received acknowledgments is commonly known as outer loop link adaptation (OLLA), in contrast to the inner loop, which consists on selecting a MCS using the margins. We assume that for each received frame the UTs feed back the outcome of the frame decoding to the gateway, which is eventually used to update the margin of each user. This variable $\epsilon_{k, t}^{[i]}$ takes the value 1 to indicate that an error has occurred at decoding of the frame number $t$ for the $i$-th user in the $k$-th beam (NAK), and it takes the value 0 otherwise (ACK). We should note that the ACK/NAK feedback is used only for adapting the margins of the link adaptation algorithms, without any retransmission of the frames at the physical layer due to the long propagation delay. Moreover, the burden of sending these acknowledgments is low compared with all the information a receiver should feed back in a system with precoding.

The MCS selection of frame $t$ intended for the multicast group of $M$ users in the $k$-th beam, $\mathrm{MCS}_{k, t}$, is performed by means of a Lookup Table (LUT), which receives as inputs the estimation of the UTs SINR, $S_{1} \hat{N} R_{k, t}^{[i]}$, computed by the gateway with the imperfect CSIT, and the adaptive margins of these users, $m_{k, t}^{[i]}$. The DVB-S2X framing structure 
imposes the use of a single MCS for the $M$ users whose data is multiplexed in a frame. Therefore, the UT with the worst signal quality will determine the MCS of the multicast group. Mathematically, we can express the MCS selection as

$$
\mathrm{MCS}_{k, t}=\prod\left(\min _{i} \operatorname{SINR}_{k, t}^{[i]}+m_{k, t}^{[i]}\right)
$$

where the LUT $\Pi(\cdot)$, which is depicted in Fig. 13, is a staircase function which maps SINR intervals to MCS following the performance tables of the DVB-S2X standard [2]. This function is usually referred to as the inner loop for link adaptation [23]. The reverse operation is done by means of the function $\mathrm{U}(\cdot)$, which returns the threshold SINR of a given MCS.

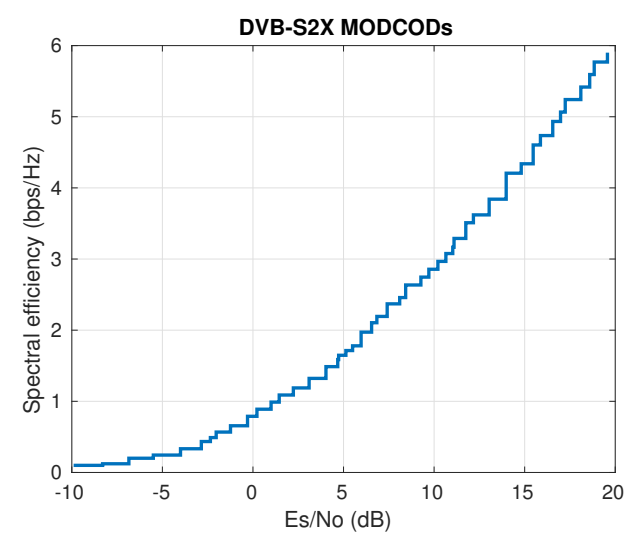

FIGURE 13 Staircase function representing the LUT of the DVB-S2X MCS, with their spectral efficiency and decoding SINR threshold.

For simulation purposes we consider that a decoding error event occurs whenever the actual SINR at the UT is lower than the SINR threshold of the MODCOD used to transmit the corresponding frame. This is expressed by means of the indicator function $[\cdot]$ as

$$
\epsilon_{k, t}^{[i]}=[\underbrace{\operatorname{SINR}_{k, t}^{[i]}}_{\text {Actual SINR }}<\underbrace{\amalg\left(\mathrm{MCS}_{k, t}\right)}_{\text {Threshold SINR of the MCS }}] .
$$

The most classic OLLA strategy consists on increasing the margin slowly $\Delta_{a c k}=\mu \cdot p_{0}$ each time an ACK is received and decreasing it more drastically, $\Delta_{n a k}=\mu \cdot(1-p 0)$, when an error occurs [23]. If the ratio between up and down steps equals $\Delta_{a c k} / \Delta_{n a k}=p_{0} /\left(1-p_{0}\right)$, a target FER of $p_{0}$ can be guaranteed. Moreover, there is a trade-off when choosing the step size: a high value of $\mu$ will increase the convergence speed of the margin at the expense of a significant oscillation at the steady state. In [9] the authors tried to solve this trade-off by proposing a new OLLA which ensures a fast convergence after a transient, occurring for example after a change in the SINR error behavior, while maintaining a good performance during steady state with infrequent margin updates. Hereafter, we provide a brief summary of this enhanced OLLA algorithm, which will be used in the simulations described in the next subsection.

The purpose of this OLLA algorithm is to maintain the FER inside the range $\left[p_{1}, p_{2}\right]$, aiming at having a target FER of $p_{0}$. The transmitter (the gateway in our case) should keep an estimation of the instantaneous FER of each 
user, which is calculated with the acknowledgments of the UTs. If FER $\ll p_{1}$ or FER $\gg p_{2}$, then the classic OLLA controller described previously is used to update the margin. The steps have a moderate size in order to increase the convergence speed and arrive soon to a situation where the FER is closed to the target value $p_{0}$.

When the FER does not take extreme values (far below $p_{1}$ or far above $p_{2}$ ), a sequential hypothesis testing (SHT) is performed and the margin is updated only if its results are conclusive. If hypothesis $\mathrm{HO}$ is true ( $\left.\mathrm{FER}<p_{1}\right)$, the margin is increased by a quantity $\lambda \cdot p_{0} \cdot N_{a c k}$. On the other hand, if hypothesis $\mathrm{H} 1$ is true (FER $>p_{2}$ ), the margin is decreased $\lambda \cdot\left(1-p_{0}\right) \cdot N_{\text {nak }}$. Table 3 collects the values of the parameters of OLLA algorithm used in our simulations. The values of the parameters are similar to those used in the original publication of the algorithm [9]. The adaptation step $\mu$ for the classic OLLA is relatively large in order to provide a fast convergence when the FER is far from the target value, whereas the adaptation step $\lambda$ used in the SHT is smaller to provide little variations in steady state. The parameter $N_{\text {nak }}$ represents the minimum number of NAKs required to accept the hypothesis H1 (FER higher than $p_{2}$ ).

\begin{tabular}{l|c} 
Parameter & Value \\
\hline Target FER $p_{0}$ & $10^{-1}, 10^{-2}, 10^{-3}, 10^{-4}$ or $10^{-5}$ \\
\hline Desired FER range $\left[p_{1}, p_{2}\right]$ & {$\left[0.9 \cdot p_{0}, 1.1 \cdot p_{0}\right]$} \\
\hline Classic OLLA range of use & FER $\leq 0.1 \cdot p_{0}$ or FER $\geq 10 \cdot p_{0}$ \\
\hline$\mu$ & 0.05 \\
$\lambda$ & 0.01 \\
\hline$N_{\text {nak }}$ & 3 \\
\hline$N_{\text {ack }}$ & $N_{\text {nak }} \cdot(1-T) / T^{1}$ \\
\hline Initial margin & 0
\end{tabular}

TAB LE 3 OLLA algorithm parameters.

This algorithm entails a relatively low complexity if compared with that of the other tasks that a gateway must perform. All the required mathematical operations are very simple (just a few sums and products), with the complexity scaling linearly with the number of outer loops (the number of users in the system). For each loop, only three variables need to be stored: the value of the margin, the estimation of the FER and the internal state of the SHT controller.

A key point is the margin initialization when a UT gets connected. Ideally, it should be near the stationary margin (if any) to avoid long underperforming transient periods. However, the derivation of the optimum margin is not an easy task since it depends on the Probability Density Function (PDF) of the SINR, the PDF of the SINR error and the MCS SINR thresholds in the UT SINR region. In an ideal system with infinite MCS, which can be emulated in practice using a very large number of MCS very close to each other in terms of SINR thresholds, it can be tested experimentally that the optimum margin depends only on the statistics of the SINR error. Namely, for a target FER of $p_{0}$, the optimum margin is $-m^{*}$ if $\mathbb{P}\left(e_{k}^{[i]}>m^{*}\right)=p_{0}$. However, in a practical DVB-S2X-based system, the threshold SINRs of the MCS are not equally separated; the distance among contiguous MCS is in the range of the SINR error magnitude, which can span several MCSs, as shown in Fig. 11. Thus, predicting the optimum margin for a specific user is not straightforward.

The DVB-S2X standard [2, Annex D.5] considers that the receiver should feed back the measured precoded SINR (therein referred as CNI). This SINR reported by the UTs, along with the estimation of the users SINR, SINRR ${ }_{k}^{[i]}$, performed by the gateway previously to MCS allocation, allows to calculate the SINR absolute error $e_{k}^{[i]}$. Therefore,

\footnotetext{
${ }^{1}$ See [9] for $T$ definition.
} 


\begin{tabular}{l|c|c|c|c|c} 
& Target $p_{0}=10^{-1}$ & Target $p_{0}=10^{-2}$ & Target $p_{0}=10^{-3}$ & Target $p_{0}=10^{-4}$ & Target $p_{0}=10^{-5}$ \\
\hline Without fading & 0.1038 & 0.0099 & $9.96 \cdot 10^{-4}$ & $1.05 \cdot 10^{-4}$ & $9.00 \cdot 10^{-6}$ \\
\hline Rice factor $K=25 \mathrm{~dB}$ & 0.1046 & 0.0108 & $1.00 \cdot 10^{-3}$ & $1.02 \cdot 10^{-4}$ & $1.08 \cdot 10^{-5}$ \\
\hline Rice factor $K=34 \mathrm{~dB}$ & 0.1038 & 0.0100 & $9.76 \cdot 10^{-4}$ & $1.05 \cdot 10^{-4}$ & $1.12 \cdot 10^{-5}$
\end{tabular}

TAB LE 4 Average FER of the 10 users with the worse channel conditions of the 10 multicast groups of the ACM simulations.

the gateway can obtain some basic statistics of both precoded SINR and SINR error after a not large number of frames. As possible future work, some supervised Machine Learning (ML) technique [24] could be used to perform a margin re-initialization after some transitory happens, avoiding long convergence times. Since the gateway manages a high number of connections, it can use information such as the margins of connections which comply with the FER constraint and their SINR and SINR error statistics, to train a neural network, for example, and use it to decide the right margin of other connections.

\section{2 $\quad$ ACM simulations results}

We have performed ACM simulations of 10 fixed multicast groups of $M=2$ users for a complete system with $N=245$ beams. Each multicast group belongs to a different beam, and the 10 beams are located far apart from each other. In the scheduling algorithm $S=6$ circular sectors have been chosen for the inter-beam scheduling, following Section 2.4. In the remaining 235 beams, random multicast groups are scheduled, with all the multicast groups belonging to an analogous circular sector in their respective beams. With this, a random variation of the SINR and the SINR error as shown in Fig. 11 occurs. The transmission of $F$ frames is simulated, with $F$ equal to 1, 000, 5, 000, 50, 000, 500, 000 and 1,000,000 for the target FERs of $10^{-1}, 10^{-2}, 10^{-3}, 10^{-4}$ and $10^{-5}$, respectively. The set of MCS, taken from the DVB-S2X standard, can be seen in Fig. 13. For MCS selection purposes, the enhanced outer and inner link adaptation algorithms detailed in the previous subsection for MCS were used. At the end of each test the experimental FER, evaluated during the last half of the simulation, is obtained.

Three different types of simulations were performed. In the first one, the CSIT degradation is only due to the real nullification and CSI estimation errors, as explained in Section 3 (the fading coefficients $f_{m m}$, which are the diagonal elements of the matrix $\mathbf{F}^{[i]}$ in (2), are equal to 1). In the other two, Rician fading is added, with Rice factors of 25 and $34 \mathrm{~dB}$, for modeling the terrestrial and aeronautical channels, respectively, following [25] and [2]. The Rice factor of $25 \mathrm{~dB}$ corresponds to a land mobile satellite channel with a directive antenna, whereas the Rice factor of $34 \mathrm{~dB}$, corresponding to the aeronautical channel, has a multipath component barely noticeable. We consider that the realizations of the fading coefficients of different users and frames are uncorrelated. As worst case, the fading coefficients $f_{m m}$ employed by the gateway to compute the estimated SINR when using $\hat{H}$ are uncorrelated with the fading coefficient $f_{m m}$ of the final actual UT SINR, present in $\mathbf{H}$, in order to take into account the effect of the delay in the CSI report.

Table 4 shows the mean FER of the simulations, obtained after averaging the experimental FER of 10 users of the simulations. It can be seen that in all cases, with and without fading, it is very close to the target value. Moreover, the FER of the users ranges typically between $80 \%$ and $120 \%$ of the target FER $p_{0}$. We should note that, since the multicast groups are fixed during all the simulations and share the same MCS, the user with the lowest SINR achieves the target FER of approximately $p_{0}$, whereas the user with the highest SINR has a much lower FER, usually close to 0. When both users of the multicast group have similar SINRs, in both cases the experimental FER is around the target value. 


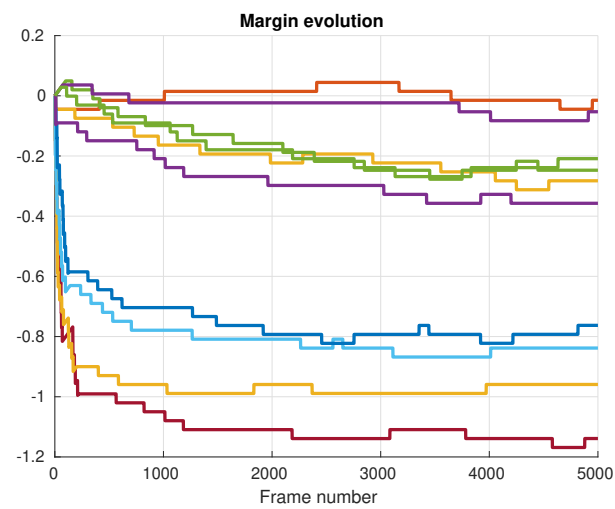

(a) Target FER 0.01

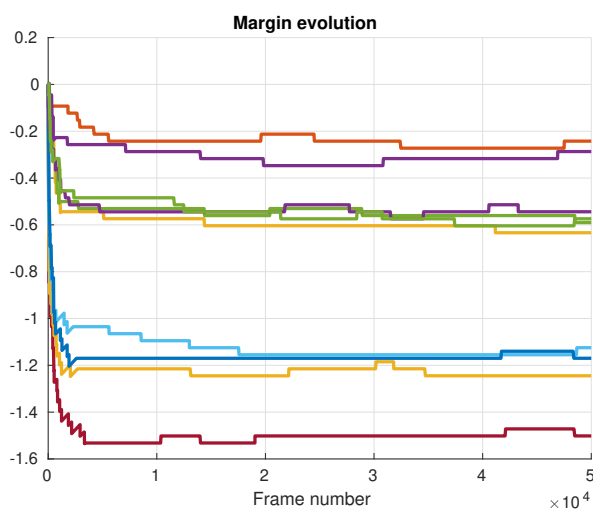

(b) Target FER 0.001

FIGURE 14 Margin evolution for the users with the lowest SINR of each multicast group (simulations with Rician fading with Rice factor $K=25 \mathrm{~dB}$ ).

Now, Fig. 14 shows the evolution of the margin for those users with the lowest SINR in each multicast group, the ones whose experimental FER matches the target FER. The back-off margins are more conservative in those cases with a lower target FER, as can be noticed in Fig. 14. The different behaviour of the link adaptation algorithm depending on the measured FER can be also observed in Fig. 14b. Initially, the classic OLLA is active, updating the margin with each received acknowledgement, and using high steps in order to achieve a fast convergence; eventually, SHT comes into play so that the margin update becomes less frequent, and only conclusive hypothesis tests trigger a margin change.

Finally, we would like to comment on the influence of the number of multicast users $M$ per frame. In our simulations the multicast groups were kept fixed from the onset; thus, for $M=2$, one of the users is always the strong one (it has the highest SNR), whereas the other is always the weak user (it has the lowest SNR). In such a case and for equal margins, the SINR of the weak user determines the MODCOD and its margin will evolve until the target FER $p_{0}$ is achieved. On the other hand, the strong user will have a FER below $p_{0}$ and its margin will evolve trying to achieve the target FER. For fixed multicast groups, as considered in this paper, and for non-fixed multicast groups with higher $M$, it would be interesting to test some modifications of the proposed algorithm. For example, it could be updated only the margin of the weakest user of the frame, and/or the thresholds of the desired FER range $\left[p_{1}, p_{2}\right]$ of Tab. 3 could be slightly modified to take into-account that the strongest user can have a FER significantly below the target value. These modifications are left as future work.

\section{8 | CONCLUSIONS}

The miss detection of interfering carriers was considered in this paper for a multibeam satellite, analysing the errors on the precoded SINR of the users which the gateway must calculate to perform MCS allocation. This leads to an increment on the rate of erroneous frames if no proper countermeasure is applied. The study of the SINR absolute error revealed that there are important differences on the maximum error among different beams, and even among different positions within the same beam. In order to ensure the robustness of the communications the use of an 
adaptive margin per user was proposed, which outperformed a global or beam-based margin in terms of throughput loss. The proposed adaptive margin helps to counteract the errors in the SINR of the users which are estimated by the gateway. This margin, independent for each UT, is updated by the gateway based on the ACK/NAK feedback from the users and is employed in the link adaptation for MCS selection. Simulation results showed that by using this link adaptation algorithm, a predefined target FER can be achieved, even in the presence of SINR calculation errors due to the miss detection of some entries of the CSI. It was also checked experimentally that the adaptive margin helped to guarantee the objective FER when Rician fading was added in the simulations on top of the nullification itself.

\section{conflict of interest}

The authors manifest that they do not have any conflict of interest.

\section{references}

[1] Tato A, Andrenacci S, Chatzinotas S, Mosquera C. Link Adaptation and Carriers Detection Errors in Multibeam Satellite Systems with Linear Precoding. In: 2018 9th Advanced Satellite Multimedia Systems Conference and the 15th Signal Processing for Space Communications Workshop (ASMS/SPSC); 2018. p. 1-8.

[2] Digital Video Broadcasting (DVB); Second generation framing structure, channel coding and modulation systems for Broadcasting, Interactive Services, News Gathering and other broadband satellite applications; Part 2: DVB-S2 Extensions (DVB-S2X). ETSI EN 302 307-2 V111 (2014-10);.

[3] Vidal O, Lacan J, Radzik J, Alberty E, Inigo P. Linear Precoding performance analysis in a Broadband satellite system with a 2-color dual-polarization reuse scheme. 31st AIAA International Communications Satellite Systems Conference, International Communications Satellite Systems Conferences (ICSSC), American Institute of Aeronautics and Astronautics 2013;.

[4] Christopoulos D, Chatzinotas S, Ottersten B. Multicast Multigroup Precoding and User Scheduling for Frame-Based Satellite Communications. IEEE Transactions on Wireless Communications 2015 Sept;14(9):4695-4707.

[5] Taricco G. Linear Precoding Methods for Multi-Beam Broadband Satellite Systems. In: European Wireless 2014; 20th European Wireless Conference; 2014. p. 1-6.

[6] Vazquez MA, Perez-Neira A, Christopoulos D, Chatzinotas S, Ottersten B, Arapoglou PD, et al. Precoding in Multibeam Satellite Communications: Present and Future Challenges. IEEE Wireless Communications 2016 December;23(6):8895.

[7] Vazquez MA, Shankar MRB, Kourogiorgas Cl, Arapoglou P, Icolari V, Chatzinotas S, et al. Precoding, Scheduling, and Link Adaptation in Mobile Interactive Multibeam Satellite Systems. IEEE Journal on Selected Areas in Communications 2018 May;36(5):971-980.

[8] Andrenacci S, Spano D, Christopoulos D, Chatzinotas S, Krause J, Ottersten B. Optimized link adaptation for DVB-S2X precoded waveforms based on SNIR estimation. In: 2016 50th Asilomar Conference on Signals, Systems and Computers; 2016. p. 502-506.

[9] Delgado RA, Lau K, Middleton R, Karlsson RS, Wigren T, Sun Y. Fast Convergence Outer Loop Link Adaptation with Infrequent Updates in Steady State. In: 2017 IEEE 86th Vehicular Technology Conference (VTC-Fall); 2017. p. 1-5.

[10] Montorsi G, Torino P, Colavolpe G, Ugolini A, Guidotti A, Vanelli-Coralli A, et al. Beyond DVB-S2X: a glimpse into a new air interface 201809 ; https : //www.researchgate.net/publication/338066640_BEYOND_DVB-S2X_A_GLIMPSE_INTO_ A_NEW_AIR_INTERFACE_OBJECTIVE_OF_THE_TUTORIAL (visited: 2019-01-16). 
[11] Christopoulos D, Arapoglou PD, Chatzinotas S. Linear Precoding in Multibeam SatComs: Practical Constraints. In: of Aeronautics AI, Astronautics, editors. 31st AIAA International Communications Satellite Systems Conference, International Communications Satellite Systems Conferences (ICSSC); 2013. .

[12] Vazquez MA, Shankar BMR, Kourogiorgas C, Arapoglou PD, Icolari V, Chatzinotas S, et al. Precoding, Scheduling and Link Adaptation in Mobile Interactive Multibeam Satellite Systems. IEEE Journal on Selected Areas in Communications 2018;.

[13] Jiang Y, Varanasi MK, Li J. Performance Analysis of ZF and MMSE Equalizers for MIMO Systems: An In-Depth Study of the High SNR Regime. IEEE Transactions on Information Theory 2011 April;57(4):2008-2026.

[14] Lagunas E, Andrenacci S, Chatzinotas S, Ottersten B. Cross-Layer Forward Packet Scheduling for Emerging Precoded Broadband Multibeam Satellite System. In: 2018 9th Advanced Satellite Multimedia Systems Conference and the 15th Signal Processing for Space Communications Workshop (ASMS/SPSC); 2018. p. 1-8.

[15] Guidotti A, Vanelli-Coralli A. Geographical Scheduling for Multicast Precoding in Multi-Beam Satellite Systems. In: 2018 9th Advanced Satellite Multimedia Systems Conference and the 15th Signal Processing for Space Communications Workshop (ASMS/SPSC); 2018. p. 1-8.

[16] Guidotti A, Vanelli-Coralli A. Clustering strategies for multicast precoding in multibeam satellite systems. International Journal of Satellite Communications and Networking;n/a(n/a). https://onlinelibrary.wiley.com/doi/abs/10.1002/ sat.1312.

[17] Andrenacci S, Chatzinotas S, Vanelli-Coralli A, Cioni S, Ginesi A, Ottersten B. Exploiting orthogonality in DVB-S2X through timing pre-compensation. In: 2016 8th Advanced Satellite Multimedia Systems Conference and the 14th Signal Processing for Space Communications Workshop (ASMS/SPSC); 2016. p. 1-8.

[18] Arapoglou PD, Ginesi A, Cioni S, Erl S, Clazzer F, Andrenacci S, et al. DVB-S2X-enabled Precoding for High Throughput Satellite Systems. Int J Satell Commun Netw 2016 May;34(3):439-455. https : //doi .org/10. 1002/sat.1122.

[19] Andrenacci S, Chatzinotas S, Reliable Channel State Information in Multibeam Satellite Communications; 2019. Luxembourg Patent Office, PCT/EP2019/070018, https://patentscope.wipo.int/search/en/detail.jsf?docId= W02020021001 (visited: 2020-03-18).

[20] Digital Video Broadcasting (DVB); Implementation guidelines for the second generation system for Broadcasting, Interactive Services, News Gathering and other broadband satellite applications; Part 2 - S2 Extensions (DVB-S2X). DVB Document A171-2 (2015-03);.

[21] Joroughi V, Vázquez M Pérez-Neira Al. Generalized Multicast Multibeam Precoding for Satellite Communications. IEEE Transactions on Wireless Communications 2017 Feb;16(2):952-966.

[22] Christopoulos D, Chatzinotas S, Ottersten B. Multicast Multigroup Precoding and User Scheduling for Frame-Based Satellite Communications. IEEE Transactions on Wireless Communications 2015 Sept;14(9):4695-4707.

[23] Rico-Alvariño A, Arnau J, Mosquera C. Link Adaptation in Mobile Satellite Links: Schemes for Different Degrees of CSI Knowledge. International Journal of Satellite Communications and Networking 2016;.

[24] Simeone O. A Very Brief Introduction to Machine Learning With Applications to Communication Systems. ArXiv e-prints 2018 Aug;.

[25] Fontan FP, Vazquez-Castro M, Cabado CE, Garcia JP, Kubista E. Statistical modeling of the LMS channel. IEEE Trans Veh Technol 2001;50(6):1549-1567. 